\title{
Noise and Intersymbol-Interference Properties of OTDM and ETDM Receivers
}

\author{
Martin M. Strasser, Member, IEEE, Peter J. Winzer, Member, IEEE, and Antonio Napoli
}

\begin{abstract}
We experimentally and theoretically confirm the similar performance of optical time-division demultiplexing (OTDM) and electrical time-division demultiplexing (ETDM) receivers for beat-noise limited return-to-zero signal detection. OTDM receivers show a slight sensitivity advantage, largely independent of parameters like the receiver's optical filter bandwidth or center frequency. The OTDM sensitivity benefit is caused by reduced intersymbol-interference properties of the OTDM receiver, as well as by the suppression of beat noise by the temporal demultiplexing window. The latter effect can be captured by extended expressions for the beatnoise variances presented in this letter.
\end{abstract}

Index Terms-Demultiplexing, electrical time-division multiplexing (ETDM), intersymbol interference (ISI), optical noise, optical time-division multiplexing (OTDM), receivers, signal detection.

\section{INTRODUCTION}

$\mathbf{T}$ HE PUSH for higher data rates, which has historically always left behind the development of high-speed electronics, necessitates the use of optical time-division multiplexing (OTDM) transmitters and receivers, until their economically more attractive electrical time-division multiplexed (ETDM) equivalents become available [1]. With the co-use of two different receiver technologies, the important question of intercomparability arises, i.e., the question of how closely OTDM receivers actually resemble ETDM receivers and, thus, allow system predicitons, both in terms of absolute sensitivity and in terms of robustness to performance degrading effects. This question becomes particularly striking when considering the completely different electrical receiver bandwidths within the two types of receiver.

This letter experimentally and theoretically compares a typical realization of an ETDM receiver (operating at data rate $R$ ) and an OTDM receiver (generating four optical tributaries at a reduced data rate $R / 4$ ) in the practically most relevant scenario of beat-noise limited direct detection of return-to-zero (RZ) ON-OFF keying. Throughout this letter, we focus on the different noise and intersymbol-interference (ISI) properties of OTDM and ETDM receivers. Other receiver degradations, such

Manuscript received March 11, 2003; revised August 5, 2003.

M. M. Strasser was with the Institute of Communications and Radio-Frequency Engineering, Vienna University of Technology, Vienna, Austria. $\mathrm{He}$ is now with Huber \& Suhner, 8330 Pfäffikon ZH, Switzerland (e-mail: mstrasser@hubersuhner.com).

P. J. Winzer is with Bell Laboratories, Lucent Technologies, Holmdel, NJ 07733 USA.

A. Napoli was with the Institute of Communications and Radio-Frequency Engineering, Vienna University of Technology, Vienna, Austria. He is now with the Polytechnic of Turin, Turin 10129, Italy.

Digital Object Identifier 10.1109/LPT.2003.818923

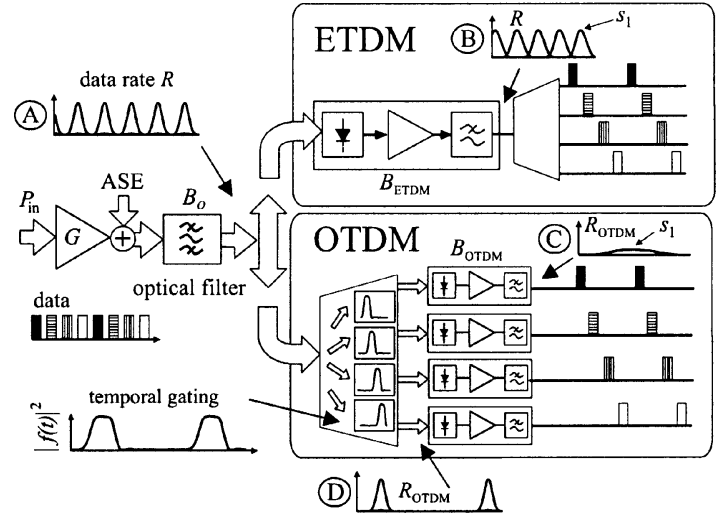

Fig. 1. Schematic setup of ETDM and OTDM receivers with corresponding optical and electrical eye diagrams.

as crosstalk between OTDM tributaries or the impact of demultiplexer window shapes are discussed in, e.g., [2] and [3].

Further, we present extended beat-noise variance expressions to account for beat-noise suppression due to optical demultiplexing. This suppression can become significant for typical electrical OTDM bandwidths and is influenced by the optical signal-to-noise ratio (OSNR).

\section{RECEIVER SETUPS}

Fig. 1 shows the schematic setup of both receiver types. An RZ signal at data rate $R$, corrupted by amplified spontaneous emission (ASE) introduced both along the transmission path and by optical preamplification, is optically filtered in order to select a channel in a wavelength-division multiplexed transmission scenario and to suppress out-of-band ASE. Detection is accomplished either by an ETDM or by an OTDM receiver. The ETDM receiver (Fig. 1, top) directly detects the filtered data stream using an overall electrical detection bandwidth $B_{\text {ETDM }}$ typically between $0.6 R$ and $0.8 R$. The signal is then electrically demultiplexed for further processing. The OTDM receiver (Fig. 1, bottom) generates four tributaries by temporally gating the optical input signal. Optical demultiplexing results in $K$ parallel data streams, each at a reduced rate of $R_{\text {OTDM }}=R / K$. These have to be detected using receivers with bandwidths $B_{\text {OTDM }} \approx B_{\text {ETDM }} / K$ only. Throughout this letter, we restrict ourselves to the most frequently encountered case of $K=4$.

In our experiments, we used a data rate of $10 \mathrm{~Gb} / \mathrm{s}$ (ETDM) and $4 \times 2.5 \mathrm{~Gb} / \mathrm{s}(\mathrm{OTDM})$. These data rates allowed for good control of the experimental parameters. The common input signal to both receiver types was a pseudorandom $45 \%$ duty cycle $\mathrm{RZ}$ bit sequence of length $2^{20}-1$, generated by a $\mathrm{LiNbO}_{3}$ 


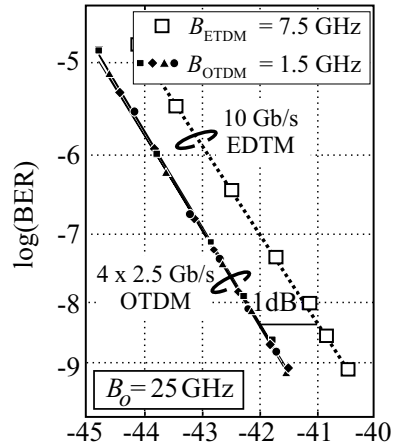

(a) input power $P_{\text {in }}[\mathrm{dBm}]$

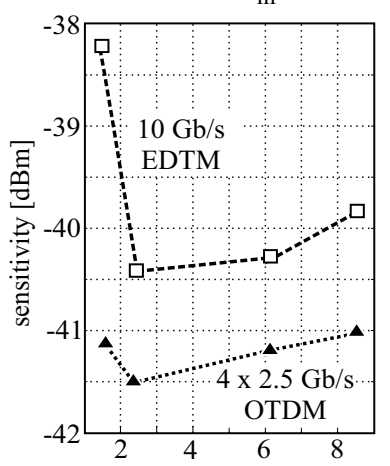

(c) optical filter bandwidth $[R]$
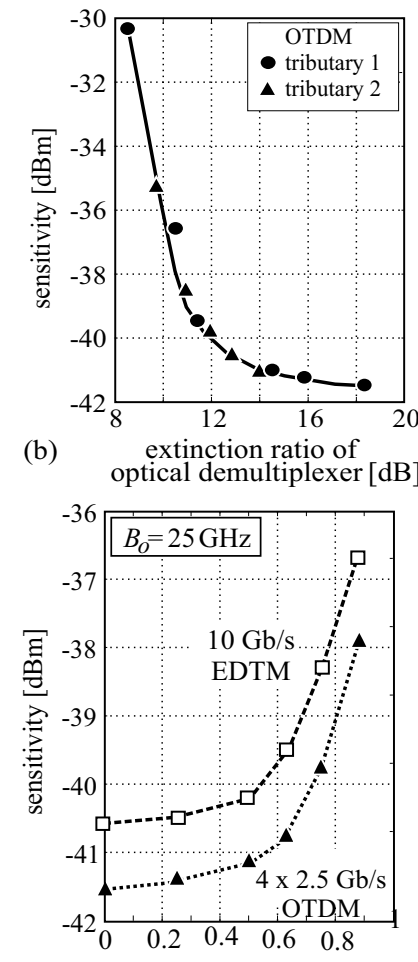

(d)

Fig. 2. (a) BER versus input power for $10-\mathrm{Gb} / \mathrm{s}$ ETDM and $4 \times 2.5 \mathrm{~Gb} / \mathrm{s}$ OTDM. (b) Influence of OTDM-demultiplexer extinction ratio. (c) Receiver sensitivity as function of optical filter bandwidth $B_{o}$. (d) Degradation due to filter center frequency offset for $B_{o}=2.5 R$.

Mach-Zehnder modulator (MZM) for data modulation and a 10-GHz sinusoidally single-arm driven $\mathrm{LiNbO}_{3}$ MZM for pulse carving. The data extinction ratio was $14 \mathrm{~dB}$. An erbium-doped fiber amplifier [(EDFA) 38-dB gain, 3.3-dB noise figure] was used as an optical preamplifier at a wavelength of $1554.94 \mathrm{~nm}$, followed by fiber Bragg gratings of various bandwidths $B_{0}$. The optical filter was followed by a second well-saturated EDFA and a 0.5-nm Fabry-Pérot filter. The second optical amplification stage made up for the insertion loss of the OTDM temporal gate and assured constant optical power at the photodetector. The temporal gate was implemented by driving an electroabsorption modulator with nearly rectangular electrical signals of $25 \%$ duty cycle. This way, four tributaries, each at a reduced data rate of $2.5 \mathrm{~Gb} / \mathrm{s}$, were generated. The maximum extinction ratio of the demultiplexing window was $18 \mathrm{~dB}$. For all our ETDM measurements, the temporal gate was set to transparency. Optoelectronic conversion was performed, for both receivers alike, by a wide-band $(40 \mathrm{GHz})$ photodiode, followed by different fifth-order Bessel filters to emulate limited receiver bandwidths $B_{\mathrm{ETDM}}=7.5 \mathrm{GHz}=0.75 R$ and $B_{\text {OTDM }}=1.5 \mathrm{GHz}=0.6 R_{\mathrm{OTDM}}$. Photodetection was verified to be beat-noise limited by measuring the receiver's thermal noise variance, as well as the noise in the presence and in the absence of an optical signal.

\section{EXPERIMENTAL RESULTS}

Fig. 2(a) shows the measured bit-error ratio (BER) as a function of the optical preamplifier input power for an optical band-

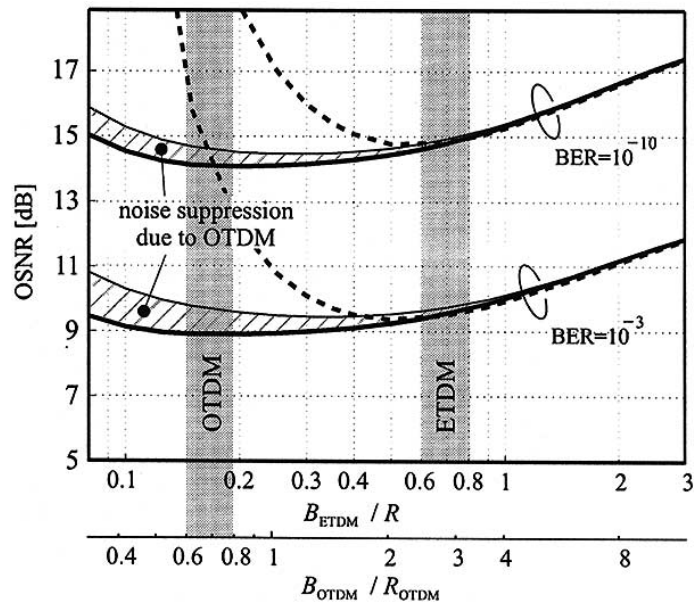

Fig. 3. Simulated performance of the ETDM receiver (dashed lines) and OTDM receiver (solid lines) in terms of required OSNR at BER $=10^{-10}$ and $\mathrm{BER}=10^{-3}$

width of $B_{o}=2.5 R$. Equal performance of all four OTDM tributaries was confirmed. The sensitivity difference between the OTDM and the ETDM receiver amounts to about $1 \mathrm{~dB}$ at a BER of $=10^{-9}$. To further characterize our OTDM receiver, we degraded the extinction ratio of the optical demuliplexer [see Fig. 2(b)], since this parameter is well known to have a significant impact on OTDM receiver performance [2], [3]. We observe a 1-dB performance degradation at an extinction ratio of $13 \mathrm{~dB}$.

Fig. 2(c) gives the measured receiver sensitivities at BER = $10^{-9}$ as a function of the optical filter bandwidth $B_{o}$, normalized to the data rate. The 1-dB sensitivity difference applies over a wide range of $B_{o}$, only growing up to $3 \mathrm{~dB}$ for very narrow $\left(B_{o}=1.5 R\right)$ optical filtering. Fig. 2(d) shows the measured sensitivities for an offset of the optical bandpass center frequency from the carrier wavelength for $B_{o}=2.5 R$. The difference between OTDM and ETDM receivers stays at $\sim 1 \mathrm{~dB}$ within the entire measurement range.

\section{THEORY AND SIMULATION}

To assess ETDM receiver performance by simulation, we used the semianalytical method detailed in [4]: We evaluated the BER, both for a single optical pulse (i.e., without the effect of ISI), as well as for a pseudorandom bit sequence of length $2^{7}-1$ (i.e., fully accounting for ISI) using Gaussian detection statistics and exact expressions for mean and variance of the electrical signal at the decision gate. We assumed 10-Gb/s RZ pulses with a duty cycle of $50 \%$, perfect data extinction, a third-order Gaussian optical filter of bandwidth $8 R$, and an electrical first-order resistance-capacitance lowpass. Our simulation results are expressed in terms of the required OSNR to obtain BER $=10^{-10}$ (Fig. 3, upper dashed curve) and BER $=10^{-3}$ (Fig. 3, lower dashed curve). The OSNR is defined as the ratio of average signal power in front of the receiver's optical filter to (unpolarized) ASE power in a 12.5-GHz optical bandwidth. At electrical bandwidths below $\sim 0.6 R$, the sensitivity starts to decrease due to the onset of electrical ISI [4], [5]. 
Due to temporal demultiplexing in the optical regime, the optical pulses of the OTDM tributaries are temporally separated by three bit slots (see Fig. 1, eye diagram (D)). As a consequence, ISI induced by postdetection electrical filtering has much less impact. Predicting OTDM receiver performance by means of the conventional ETDM beat-noise expressions [4] (Fig. 3, thin solid lines), we therefore, closely reproduce the single-pulse case of the ETDM receiver. Only at electrical detection bandwidths $B_{\mathrm{OTDM}}$ smaller than $\sim 0.6 R_{\text {OTDM }}=0.15 R$, ISI sets in and receiver performance starts to degrade. The similar ISI-free performance at typical electrical bandwidths of OTDM and ETDM receivers (see Fig. 1, gray areas) can be intuitively understood as follows: Due to the narrow detection bandwidth of the OTDM receiver, the tributaries' pulses are spread in time over four bit slots. At the same time, the maximum pulse height $\left(s_{1}\right)$ is reduced by a factor of about four compared to its value for the ETDM receiver (cf. Fig. 1, eye diagrams (B) and (C)). This reduction in electrical pulse amplitude makes up for the noise reduction due to narrower electrical filtering, and lets receiver performance become largely independent of the electrical bandwidth (and, thus, of the receiver type) in the ISI-free case [6].

In the above discussion, as well as in obtaining the thin solid line for the OTDM receiver in Fig. 3, we have assumed that the same beat-noise formulae are valid for OTDM and ETDM receivers. However, performing a more rigorous analysis for the OTDM receiver, paralleling the derivation in [6] but taking into account the cyclo-stationarity of temporally gated ASE, we arrive at the signal-ASE beat-noise expression

$$
\begin{aligned}
\sigma_{\mathrm{s}-\mathrm{ASE}}^{2}(t)= & 2 C^{2} \iint_{-\infty}^{+\infty} \operatorname{Re}\left\{e(\tau) e^{*}(\tilde{\tau})\left\langle\mathbf{n}^{*}(\tau) \mathbf{n}(\tilde{\tau})\right\rangle\right\} \\
& \times|f(\tau)|^{2}|f(\tilde{\tau})|^{2} h(t-\tau) h(t-\tilde{\tau}) d \tau d \tilde{\tau}
\end{aligned}
$$

and at the ASE-ASE beat-noise expression

$$
\begin{aligned}
\sigma_{\mathrm{ASE}-\mathrm{ASE}}^{2}(t)=M C^{2} \cdot \iint_{-\infty}^{+\infty} \mid & \left.\left|\mathbf{n}^{*}(\tau) \mathbf{n}(\tilde{\tau})\right\rangle\right|^{2}|f(\tau)|^{2}|f(\tilde{\tau})|^{2} \\
& \times h(t-\tau) h(t-\tilde{\tau}) d \tau d \tilde{\tau} .
\end{aligned}
$$

In these expressions, $C$ denotes the overall optoelectonic conversion factor, $M$ specifies the number of ASE (polarization) modes, and $e(t)$ stands for the optical signal field at the input to the temporal gate, whose (field) windowing function is denoted $f(t)$ (i.e., it multiplies the input optical power by $|f(t)|^{2}$ ). The function $\left\langle\mathbf{n}^{*}(\tau) \mathbf{n}(\tilde{\tau})\right\rangle$ denotes the autocorrelation of the stationary, optically filtered ASE before demultiplexing, and $h(t)$ is the impulse response of the entire opto-electronic detection chain.

In analyzing the above expressions, note first that we recover the conventional signal-ASE beat-noise term for the ETDM receiver [6] from (1) by assuming a rectangular gating function $f(t)$ that passes the entire optical pulse. For other gating func- tions, (1) means that both $e(t)$ and $n(t)$ enter the beat-noise integral after multiplication with $f(t)$, which indeed represents the underlying physical process. As for the ASE-ASE beatnoise term, we first notice a time-dependence as a consequence of temporal gating, i.e., ASE-ASE beat noise is nonstationary in an OTDM receiver. We also see from (2) that the effect of ASE-ASE beat noise on detection is reduced by OTDM reception, since ASE can only reach the detector for a fraction of the tributaries' bit duration, determined by $f(t)$. Note that the relative importance of ASE-ASE beat noise over signal-ASE beat noise increases with decreasing electrical detection bandwidth. Thus, the sensitivity of the OTDM receiver somewhat improves at low $B_{\mathrm{OTDM}}$, as captured by the thick solid lines in Fig. 3, which are based on (1) and (2). The OTDM performance enhancement due to suppression of ASE-ASE beat noise-visualized by the hatched area in Fig. 3-depends on the electrical filter shape and bandwidth, and becomes noticeable for typical electrical OTDM bandwidths. Further, the ASE-ASE beat-noise suppression depends on the OSNR (cf. Fig. 3: upper curves for BER $=10^{-10}$, lower curves for BER $=10^{-3}$ ), since the ASE-ASE beat noise increases quadratically with reduced OSNR, as compared to a linear growth of signal-ASE beat noise with reduced OSNR. However, this does not necessarily mean that the difference between OTDM and ETDM becomes larger at low OSNR since we also find reduced ISI penalties at low OSNR. The reason for the slight performance difference between OTDM and ETDM receivers decomposes into different amounts of ISI and different beat-noise variances.

Summarizing, our simulations and experiments confirm that OTDM receivers can perform slightly better than ETDM receivers, which can be attributed to the difference in electrical ISI as well as to ASE-ASE beat-noise suppression due to optical demultiplexing. The performance difference between the two types of receivers is found to be largely independent of the choice of the optical receiver bandwidth, and is hardly affected by an offset of the filter's center frequency.

\section{REFERENCES}

[1] R.-J. Essiambre, G. Raybon, and B. Mikkelsen, "Pseudo-linear transmission of high-speed TDM signals: 40 and $160 \mathrm{~Gb} / \mathrm{s}$," in Optical Fiber Telecommunications, I. Kaminov and T. Li, Eds. New York: Academic, 2002, vol. IV B.

[2] J. Zhang, M. Yao, X. Chen, L. Xu, M. Chen, and Y. Gao, "Bit error rate analysis of OTDM system based on moment generation function," $J$. Lightwave Technol., vol. 18, pp. 1513-1518, Nov. 2000.

[3] G. Toptchiyski, S. Randel, K. Petermann, C. Schubert, J. Berger, and H. G. Weber, "Characterization of switching windows of an $160-\mathrm{Gb} / \mathrm{s}$ all-optical demultiplexer with data base rates of 10 and $40 \mathrm{~Gb} / \mathrm{s}$," IEEE Photon. Technol. Lett., vol. 14, pp. 534-536, Apr. 2002.

[4] P. J. Winzer, M. Pfennigbauer, M. M. Strasser, and W. R. Leeb, "Optimum filter bandwidths for optically preamplified NRZ and RZ receivers," J. Lightwave Technol., vol. 19, pp. 1263-1273, Sept. 2001.

[5] M. M. Strasser, M. Pfennigbauer, M. Pauer, and P. J. Winzer, "Experimental verification of optimum filter bandwidths in direct-detection (N)RZ receivers limited by optical noise," in Proc. LEOS Annu. Meeting (LEOS 2001), 2001, Paper WK5, pp. 485-486.

[6] P. J. Winzer and A. Kalmar, "Sensitivity enhancement of optical receivers by impulsive coding," J. Lightwave Technol., vol. 17, pp. 171-177, Feb. 1999 